Supporting Information:

\title{
"Keto-Enol Tautomerization as a First Step in Hydrogenation of Carbonyl Compounds"
}

Smadar Attia, Marvin C. Schmidt, Carsten Schröder, Jann Weber, Ann-Katrin Baumann and Swetlana Schauermann*

Institute of Physical Chemistry, Christian-Albrechts-University Kiel, Max-Eyth-Str. 2, 24118, Kiel, Germany

Corresponding author: schauermann@pctc.uni-kiel.de

\section{Experimental Details}

All experiments have been performed at the Christian-Albrechts-University, Kiel, Germany. The molecular beam/IRAS experiments were performed in an UHV apparatus (base pressure $2 \cdot 10^{-10}$ mbar) containing two effusive doubly differentially pumped multi-channel array source (indicated in the manuscript as effusive beams) and a supersonic beam operated at room temperature. Further details on the apparatus can be found elsewhere. ${ }^{1}$

Acetophenone (Sigma-Aldrich, purity 99\%) was dosed onto the sample via an effusive source at a typical flux of $2 \cdot 10^{12}$ molecules $\cdot \mathrm{cm}^{-2} \cdot \mathrm{s}^{-1}$ controlled by a pneumatic valve and a shutter. The source was operated at room temperature, and the beam diameter was chosen to exceed the sample size. The $\operatorname{Pt}(111)$ single crystal (MaTeck $\mathrm{GmbH}$ ) was cleaned prior to use by repeated cycles of $\mathrm{Ar}+$ ion bombardment at room temperature, followed by oxidation in $1 \cdot 10^{-6} \mathrm{mbar}_{2}$ at $750-800 \mathrm{~K}$ to remove residual carbon and subsequent annealing at $1150 \mathrm{~K}$. The last cleaning cycle contained reduction of the surface with $1 \cdot 10^{-6} \mathrm{mbar} \mathrm{CO}$ at $400-450 \mathrm{~K}$. Shortly before each experiment the sample was flashed to $600 \mathrm{~K}$ before cooling to the required temperature to remove $\mathrm{CO}$ adsorbates. The flatness and cleanliness of the Pt(111) single-crystal were checked by Low Energy Electron Diffraction (LEED), Auger Electron Spectroscopy (AES) and additionally by IRAS of adsorbed $\mathrm{CO}$ to probe the abundance of adsorption sites. Acetophenone was purified prior to the experiments by repeated freeze-pump-thaw cycles.

IRAS data have been acquired using a vacuum Fourier transform infrared (FT-IR) spectrometer (Bruker Vertex 80v) with a spectral resolution of $2 \mathrm{~cm}^{-1}$ and a mid-infrared (MIR) polarizer and p-polarized IR light. The spectrometer is equipped with a narrow band Mercury-CadmiumTelluride (MCT) detector. All IRAS spectra were acquired at the same surface temperature that was used for acetophenone deposition. An automated quadrupole mass spectrometer (QMS) system (Hiden, HAL 301/3F) was employed for the continuous monitoring of the partial pressures of gaseous species. 
The STM measurements were carried out in a different UHV (base pressure better than $1 \cdot 10^{-10}$ mbar) apparatus by employing a variable temperature (90-300 K) Aarhus 150 SPM (SPECS). All measurements were carried out in a constant current mode and the bias voltage $\mathrm{U}_{\mathrm{T}}$ was applied to the sample. The etched W-Tip was commercially produced by SPECS and in-situ sharpened by 3 $\mathrm{keV} \mathrm{Ar+} \mathrm{-bombardment} \mathrm{with} 5 \cdot 10^{-6} \mathrm{mbar}$ back pressure for $5 \mathrm{~min}$. Acetophenone was purified prior to the experiments by repeated freeze-pump-thaw cycles.

The $\operatorname{Pt}(111)$ single crystal was cleaned according to the cleaning procedure described above. The quality of the sample was additionally verified by acquiring STM images prior to the deposition. Acetophenone was dosed through a dedicated gas doser at different temperatures ranging from 150 to $300 \mathrm{~K}$ followed by imaging at the temperatures indicted in the manuscript.

\section{Temperature dependence of acetophenone oligomer formation}

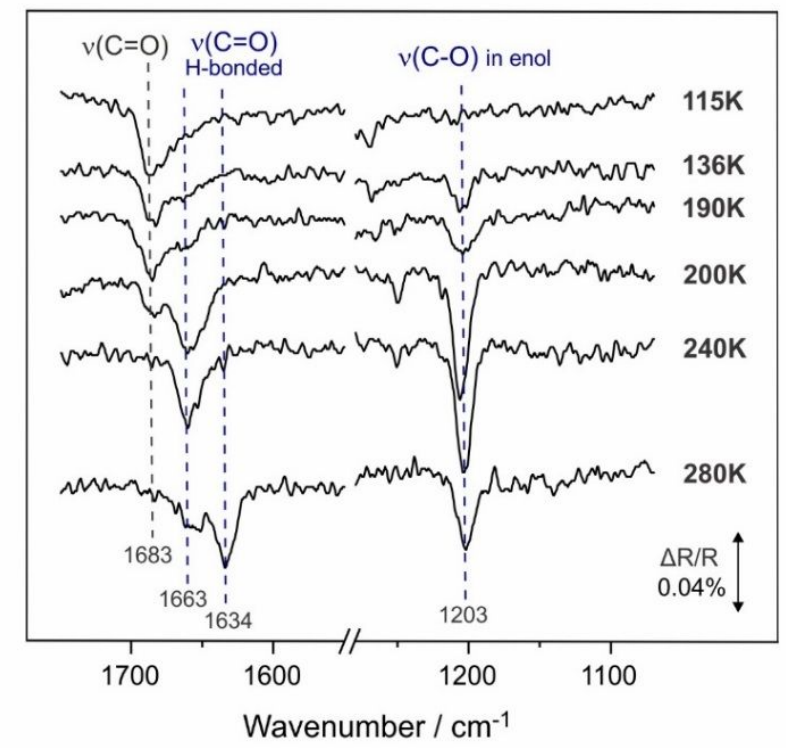

Figure S1. IR spectra of acetophenone adsorbed on $\operatorname{Pt}(111)$ at the temperatures indicated at the right side. Only the $\mathrm{C}=\mathrm{O}$ and $\mathrm{C}-\mathrm{O}$ stretching regions are shown. All spectra were obtained at submonolayer coverages after acetophenone exposures of $2.3 \cdot 10^{15}(115 \mathrm{~K}), 3.8 \cdot 10^{14}(136 \mathrm{~K}), 1.0 \cdot 10^{16}$ $(190 \mathrm{~K}) ; 6.5 \cdot 10^{14}(200 \mathrm{~K}), 4.0 \cdot 10^{14}(240 \mathrm{~K}) ; 9.0 \cdot 10^{14}(280 \mathrm{~K})$ molecules $\cdot \mathrm{cm}^{-2}($ after [2] $)$

Figure S1 shows the spectra of acetophenone on $\operatorname{Pt}(111)$ obtained in the temperature range from 115 to $280 \mathrm{~K}$ at coverages close to saturation at any given temperature. At the lowest temperature $(115 \mathrm{~K})$, only the band at $1683 \mathrm{~cm}^{-1}$ can be observed, which is related to the $\mathrm{C}=\mathrm{O}$ stretching vibrational frequency of a nearly unperturbed molecule. At this temperature, acetophenone is adsorbed only as monomer species as evidenced by STM. Starting from 136-190 K, two new bands evolve - at $1663 \mathrm{~cm}^{-1}$ and at $1203 \mathrm{~cm}^{-1}$, which can be assigned to ketone-enol dimers as discussed 
in the manuscript. These bands gain in absolute and relative intensity with increasing temperature and become the most prominent vibrational peaks in the temperature range 200-240 K. It should be emphasized that at $240 \mathrm{~K}$ the ketone-enol dimers are the only species observed spectroscopically. At the temperature $280 \mathrm{~K}$, the vibrational band at $1634 \mathrm{~cm}^{-1}$ evolves pointing to the formation of the ketone-enol-enol trimers, which co-exist with the ketone-enol dimers as suggested both by IRAS and STM. ${ }^{2}$

\section{References:}

[1] Attia, S.; Spadafora, E.J; Hartmann, J; Freund, H.-J; Schauermann, S. "Molecular Beam/ Infrared Reflection-Absorption Spectroscopy Apparatus for Probing Heterogeneously-Catalyzed Reactions on Functionalized and Nanostructured Model Surfaces. Rev. Sci. Inst, 90 (2019) 5390353917.

[2] Attia, S.; Schmidt, M. C.; Schröder, C.; Schauermann, S. „Formation and Stabilization Mechanisms of Enols on Surfaces Through Multiple Hydrogen Bonding." ACS Catalysis 9 (2019) 6882-6889. 